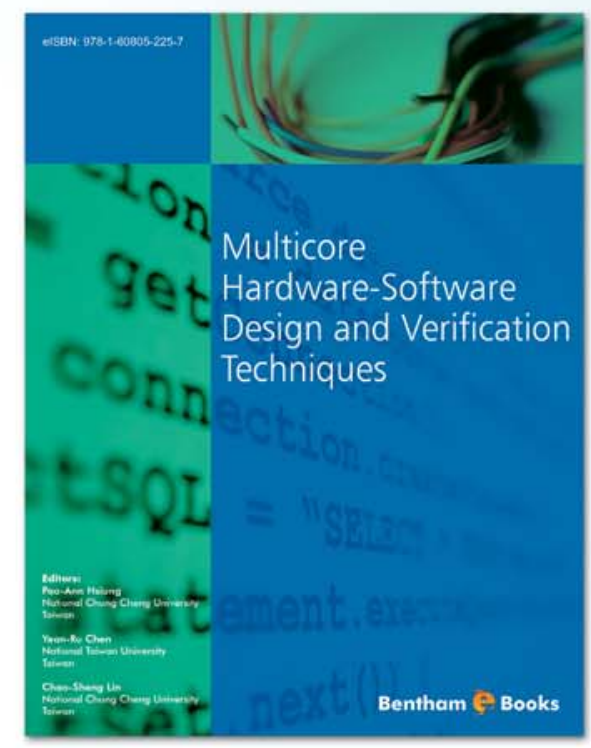

\section{Editors:}

Pao-Ann Hsiung

Taiwan

Yean-Ru Chen

Taiwan

Chao-Sheng Lin

Taiwan

elSBN: 978-1-60805-225-7

\title{
Multicore Hardware-Software Design and Verification Technicues
}

\section{wrwhenthamscience.com/ebooks/9781608052251}

\section{About the ebook}

This e-book attempts to cover the design and verification issues related to different application domains as a singular source of reference to the state-of-the-art techniques in multicore processor design and software programming that covers multiple application domains.

\section{Contents}

Acknowledgements

- Affinity and Distance-Aware Thread Scheduling and Migration in Reconfigurable Many-Core Architectures

- On the Design of Multicore Architectures Guided by a Miss Table at Level-1 and Level-2 Caches to Improve Predictability and Performance/Power Ratio

- TRoCMP: An Approach to Energy Saving for Multi-Core Systems

- Model-Driven Multi-core Embedded Software Design

- Automatic High-Level Code Generation for Multi-Core Processors in Embedded Systems

- Index

Methicillin Resistant Staphylococcus aureus (MRSA)

For Sales Advertising Inquiries: Contact: marketing@benthamscience.org 